\title{
Circuit
}

Musiques contemporaines

\section{[Sans titre]}

\section{Gerhard Stäbler}

Volume 15, numéro 3, 2005

Souvenirs de Darmstadt : retour sur la musique contemporaine du dernier demi-siècle

URI : https://id.erudit.org/iderudit/902373ar

DOI : https://doi.org/10.7202/902373ar

Aller au sommaire du numéro

\section{Éditeur(s)}

Les Presses de l'Université de Montréal

ISSN

1183-1693 (imprimé)

1488-9692 (numérique)

Découvrir la revue

Citer cet article

Stäbler, G. (2005). [Sans titre]. Circuit, 15(3), 78-81.

https://doi.org/10.7202/902373ar

\section{Résumé de l'article}

Après Darmstadt 1972 et Darmstadt 1974, au cours desquels il a assisté aux conférences de Stockhausen, Ligeti et Christian Wolff, le compositeur allemand prend ses distances vis-à-vis des préoccupations centrales des cours d'été de cette époque. Il retourne aux Ferienkurse après une absence volontaire de près de deux décennies, pendant lesquelles des questions sociales et politiques d'ordre plus concret le préoccupent davantage. Il présente alors son oeuvre multimédia pour plusieurs artistes [voix ${ }^{\text {(time) }}$ ] et découvre que le Darmstadt des années 1990 est plus réceptif à ses préoccupations sociales, ainsi qu'aux oeuvres multimédias et collectives.
Ce document est protégé par la loi sur le droit d'auteur. L'utilisation des services d'Érudit (y compris la reproduction) est assujettie à sa politique d'utilisation que vous pouvez consulter en ligne.

https://apropos.erudit.org/fr/usagers/politique-dutilisation/ 
- "Toleraws" $\rightarrow$ tablen]

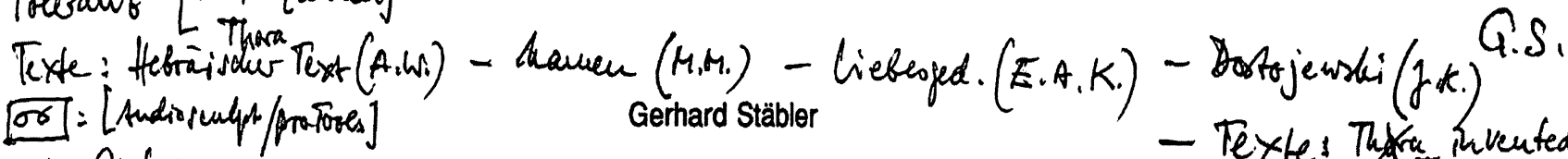

1 Allen Givistery

- Muscel auchamp

- Harry Cowrell

- [Fituess durley...]

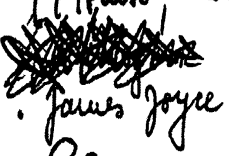

- Coge

- pectert
Basic Plan [voix(time)]

Internationale Darmstädter Ferienkurse - July / August 1998
- Textes Thxora invented $(V, K$.

(imitation)

in cooperation with

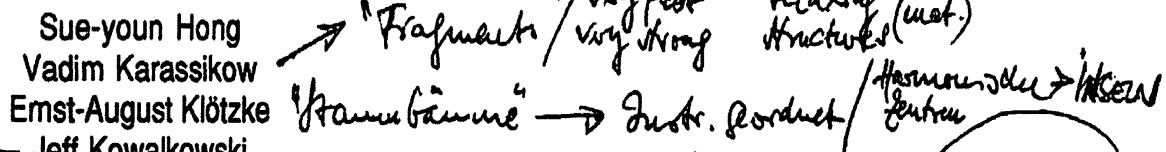

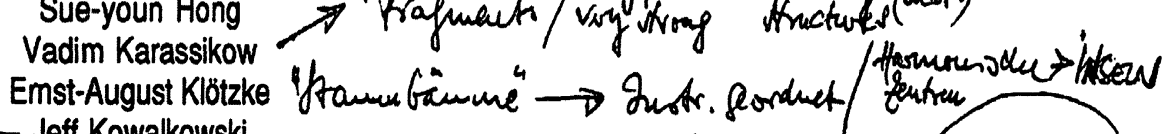

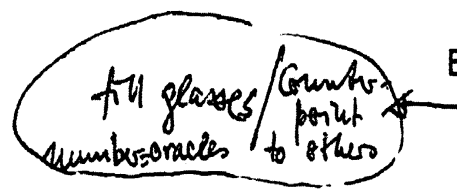

"wadelife faluetorte" $\longleftarrow$ Kunsu Shim Anounghiter - Mentratiter

$$
\text { Jeff Kowalkowski }
$$

Jeff Kowalkowski

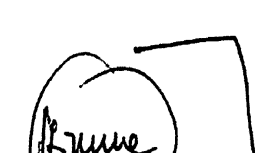

Amnon Wolman of "Muegic"

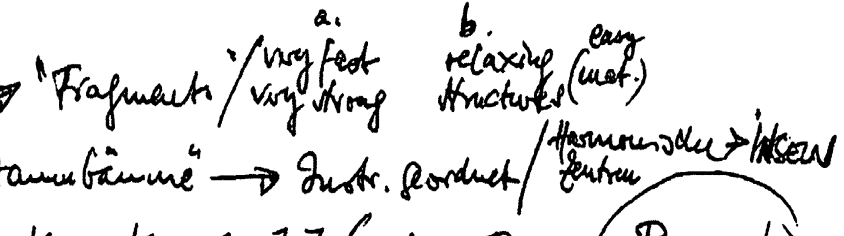

Heiko Daxl

= userinastes Dustrumens des heanch Veit-Lup

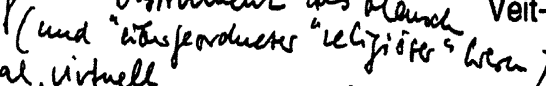

$=$ Leal, virtuell

= imere dañe (Genrser)

fereut. Texte kunst texte

fert

Ensemble belcanto, - Dietburg Spohr Ensemble Modern (Vykintas Bieliauskas, conductor)

Clunstoph liór solf Schaeter als tween S Linju Internationales Musikinstitut Darmstadt

Gerd Rische

Studio für elektroakustische Musik

Akademie der Künste Berlin

○ Gerhard Stäbler vinutererevoliafon

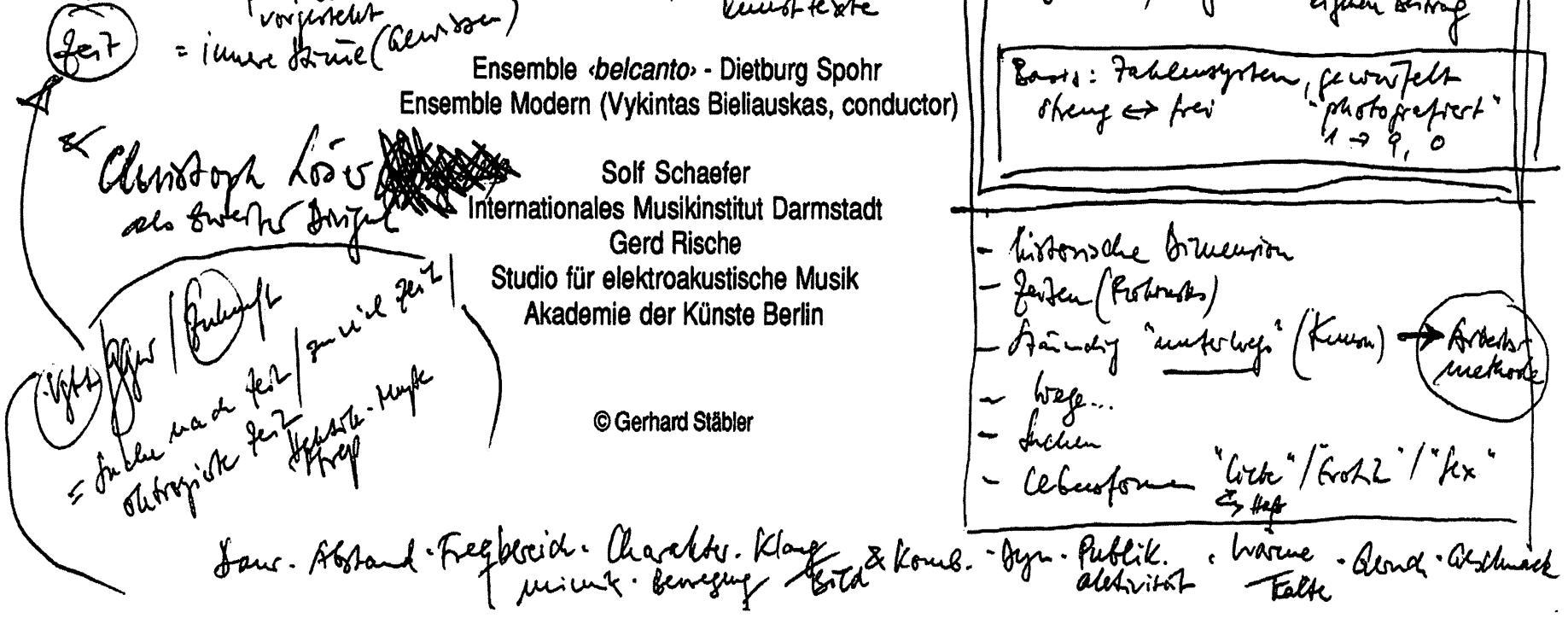

Plan de base de [voix (time)] avec esquisses de Gerhard Stäbler sur de divers aspects des différentes contributions compositionnelles et idées pour l'organisation de cette œuvre multimédia (1998). 


\section{SOUVENIRS}

Gerhard Stäbler (trad.: Yves Saint-Amant)

\section{1) Quel souvenir emblêmatique, ou du moins significatif, garderiez-vous de votre passage à Darmstadt?}

\section{2) Pour vous, que représente Darmstadt aujourd'hui?}

J'ai encore sur mes étagères des piles de notes prises en 1972 et en 1974, lors des cours donnés par György Ligeti, Karlheinz Stockhausen, Christian Wolff et plusieurs autres, des cours qui eurent des répercussions - sur le plan du contenu et sur le plan politique. C'était l'époque où je plongeai dans l'avantgarde musicale en tant que membre de l'Ensemble musica negativa de HeinzKlaus Metzger et Rainer Riehn. Mais c'était aussi l'époque où l'avant-garde fut soumise au "test de la réalité » : ce qu'on exigeait de la musique fut examiné à la lumière de l'effet produit; ses contenus furent étudiés sous l'angle de leur pertinence. J'étais fasciné par la cohérence tant chez Ligeti que chez Stockhausen et Wolff, une cohérence colorée par le style de chacun. Je ramenai chez moi l'examen approfondi du matériau musical (Stockhausen), la remise en question de son essence (Metzger), l'ouverture théoriquement possible sur des questions sociales, voire politiques (Schnebel, Wolff), ainsi que l'exigence d'une musique revendicative (Nono, Lachenmann, Huber). Mais l'exclusivité (ressentie ou réelle?) ne m'intéressait pas... Et mon scepticisme à l'égard d'une révolution matérielle et immanente, présentée comme un point 
de départ pour changer la société, grandit à ce point que je renonçai à la composition pour un temps.

Bien que le début des années 1980 ait confirmé cet état de choses, mes tentatives réitérées de composition me conduisirent dans des domaines captivants. Après des années passées à travailler avec des troupes de théâtre de rue et à la mise sur pied du magazine culturel Linkskurve, je me sentais bien à l'extérieur des salles de concerts habituelles - parce que cela m'incitait à aiguiser mes sens, tous mes sens. D'anciennes mines, des ateliers d'usines abandonnées, des entrepôts désaffectés fournissaient l'ambiance, les lieux propices à une confrontation, afin de mettre la musique en contact non seulement avec les choses du quotidien qui étaient les plus urgentes, mais afin de faire d'elle une partie des questions existentielles, philosophiques, esthético-politiques posées par la vie elle-même.

Animé par un désir d'appartenance resté (encore) bien vivant malgré le fait que j'aie gardé mes distances, je partis d'Essen à l'été de 1984 expressément pour me rendre à Darmstadt. Par un après-midi de juillet particulièrement chaud et humide, j'arrivai à l'école Georg-Büchner, mais quand je regardai par la fenêtre et vis les mêmes visages, le même comportement chez les participants - c'était là un tableau qui m'était resté en mémoire après les cours suivis dix ans auparavant et qui réveillait en moi le souvenir de nombre de discussions artificielles et coupées du réel portant sur le structuralisme et le formalisme musical - , je lâchai la poignée sans avoir ouvert la porte et rentrai directement à la maison.

Y avait-il eu un changement? Quelque chose devait bien changer peu à peu car dans les années 1980, le domaine musical s'était élargi énormément. Non seulement les autres cultures, en particulier celles d'Extrême-Orient, réclamaient-elles de plus en plus notre attention, mais les médias les plus variés se répandirent et exercèrent une grande fascination sur la musique. Cage, qui avait contribué à préparer cela, se concentra, à la fin de sa vie certes, de nouveau plutôt sur le son. Dans les années 1990, l'éventail des possibilités offertes par Musik plus..., dont on doit chercher l'origine dans les nombreux bouleversements radicaux qu'ont connus les arts durant le $\mathrm{xx}^{\mathrm{e}}$ siècle, s'élargit encore et encore. C'était là un développement qui tendait de plus en plus vers des projets multimédias (entre autres [voix ${ }^{\text {(time) }}$ ], un projet que je dirigeai durant les Darmstädter Ferienkurse de 1998 et qui réunit dans une même œuvre neuf compositeurs et compositrices et trois artistes en vidéo venus du monde entier) et dont on ne peut plus se passer, pas plus que des « radiophonies " présentées ces dernières années à Darmstadt. Ce développement montre que la musique se libère de l'élitisme mais qu'elle doit par contre se fixer à elle-même des 
règles précises afin de ne pas se livrer au Design ou tout simplement se prostituer. Voilà - pour moi - une perspective extrêmement captivante, car à l'intérieur de ces coordonnées l'art (et la musique avec lui, en lui) joue un rôle essentiel, capital, celui de rendre la vie digne d'être vécue.

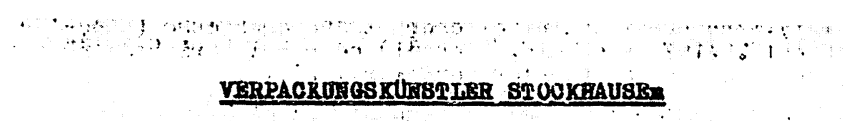

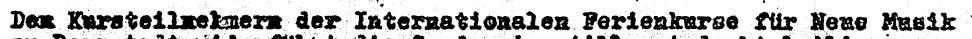

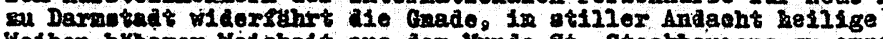

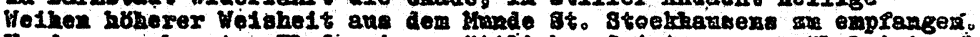

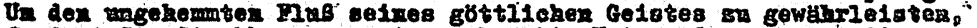
Foxlert 8t. Stookhpusen meingesolntakte Onterordiung pater dis ron

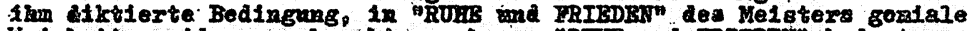

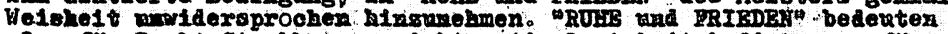
also fir Bankt Stookbataen nisht optinalo Arbeltabodininengen fur

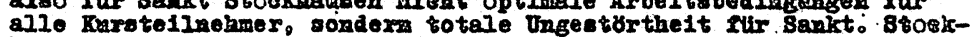

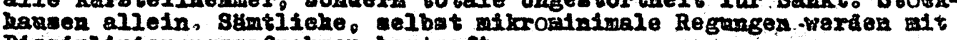
D1seipliniermeomafpalmen bestraft

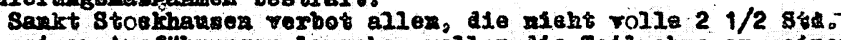
selnex Ansfluminger laxsehen wollen die Tellnawme an seirer. Seminar, So hat or beisplelowelse ar 3.8 .74 elnex flarischea

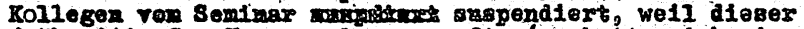

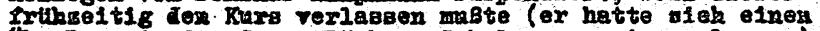
toe-Rawn in der Georg-Bllehner-Sohnle reasrvieren Iassen).

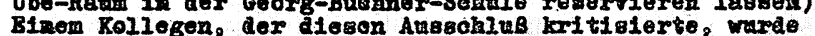

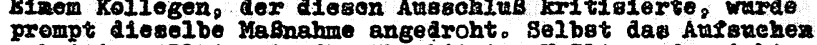
gohattiger 'Platse in der tiberhitaten HaIle wardo alehs goendets.

Die: organisatoribohen DIgsiplintercngen wihresia des seminaxs -

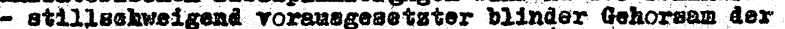

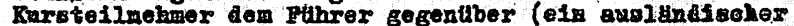

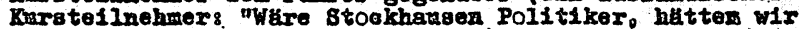
einen Diktator mahr in dex Welt." )

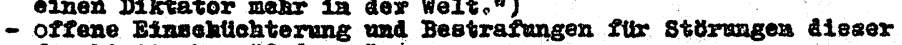

- Orfene Blabehtiohterung vo

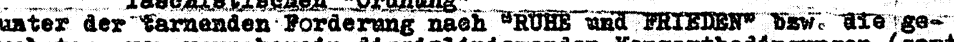
wohnton, ron vornehorein diagiplinlerenden Konsertbedingangen (eamt St. Stockhousens Predigt vor der Mraikweiho am 4.8 .74 in der 8 port.

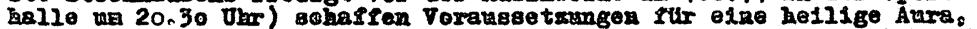
die nahosw alle avantgardistischen Korpositioner, ror allem aber.

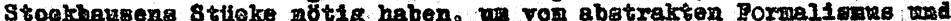

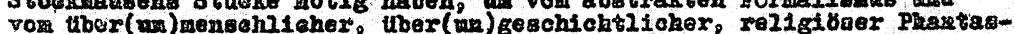

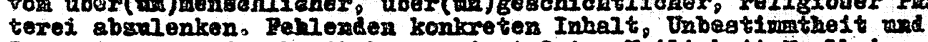

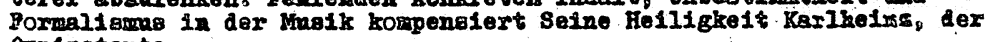
rmipotente,

- mit abotrakter Binsigartigkest

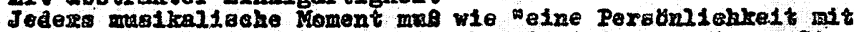
- Igenom Charakter, wie ein Mensoh, plex oder was lnmer sie willex, now kempontes't werden.

- mit toexreitilchkeit

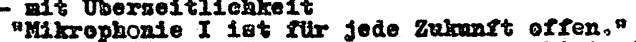

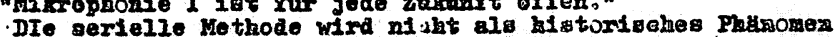

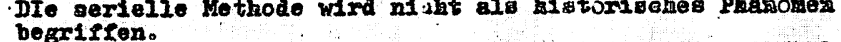

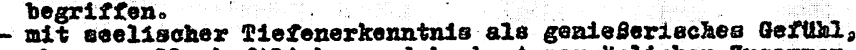
ohne gesellsohaftilohen und konkret perebnlichen Zusammexhang.

"Ba gibt kein Werk ron mis, tas so sehr dae Unterbewabtaeix

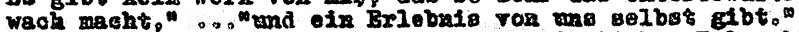

- mit der Beschwbreang ungreipberer, Ubersinnlicher Brlekshtrang dures aniverselies Bins-Sein mit dem Kosmos. ("ave Afis ateben Tagen")

"Verpackungskünstler Stockhausen", pamphlet sur l'art pédagogique de Stockhausen, distribué au cours de Darmstadt en 1974. 